\title{
Interactions of Meibomian gland secretion with polar lipids in Langmuir monolayers
}

\author{
G. As. Georgiev ${ }^{\mathrm{a}, *}$, E. Kutsarova ${ }^{\mathrm{a}}$, A. Jordanova ${ }^{\mathrm{b}}$, R. Krastev ${ }^{\mathrm{c}}$, Z. Lalchev ${ }^{\mathrm{a}}$ \\ a Department of Biochemistry, Faculty of Biology, University of Sofia, 1164 Sofia, Bulgaria \\ ${ }^{\mathrm{b}}$ Institute of Biophysics, Bulgarian Academy of Science, 1123 Sofia, Bulgaria \\ ${ }^{c}$ NMI Naturwissenschaftliches und Medizinisches Institut an der Universität Tübingen, 72770 Reutlingen and Max Planck Institute of Colloids and Interfaces, \\ 14476 Potsdam-Golm, Germany
}

\section{A R T I C L E I N F O}

\section{Article history:}

Received 8 November 2009

Received in revised form 6 March 2010

Accepted 22 March 2010

Available online 30 March 2010

\section{Keywords:}

Meibomian gland secretion

Polar lipids

Langmuir monolayers

Surface interactions

Artificial tears

\begin{abstract}
A B S T R A C T
The surface interactions of Meibomian gland secretion (MGS) with polar lipid (PL), Egg Sphingomyelin (SM) or Dipalmitoylphosphatidylcholine (DPPC), are studied in mixed pseudo-binary films formed at the air/water interface of Langmuir surface balance. The behavior of the mixed films during slow quasi-equilibrium compression and during fast dynamic compression-decompression is registered by measurements of surface pressure and surface potential, and by monitoring film morphology with Brewster Angle Microscopy (BAM). Quasi-equilibrium compression isotherms are used to calculate the excess Gibbs and Helmholtz energy of mixing between MGS and PLs and thus to evaluate the interactions between the lipid compounds at the interface. The effects of PLs on the mixed film's elastic moduli of area compressibility, morphology and capability to attain high surface pressures are also examined.

PLs interact with MGS with different strength and in different manner: MGS-SM interaction is weak and might lead to interfacial disaggregation of the thick meibium domains when SM is in excess, while MGS-DPPC interaction is strong and results in the formation of thick lipid aggregates. Both PLs increase the mixed films reciprocal compressibility and capability to achieve higher surface pressures. The results demonstrate that in vitro studies of the surface interactions between MGS and PLs might be beneficial in the selection of PLs for artificial tear formulations and for examination on molecular scale of the possible role of PLs at the ocular surface.
\end{abstract}

(c) 2010 Elsevier B.V. All rights reserved.

\section{Introduction}

Tear film (TF) is a complex lipid-protein mixture that forms $3 \mu \mathrm{m}$ thin layer over the entire (approximately $2 \mathrm{~cm}^{2}$ ) ocular surface [1]. This large area-to-volume ratio determines the importance of the low surface tension at the air/tear interface for the overall TF stability [2,3]. Key role for lowering of the surface tension plays the lipid layer that covers the tear surface. It used to be commonly accepted that the lipid layer consists of up to $10 \%$ polar lipids (mainly phospho- and sphingolipids) and of 90\% non-polar lipids (sterol esters, waxes, etc.) with both types of lipid compounds delivered entirely by the oily secretion of the Meibomian glands located in the tarsal plates of the eyelids [4,5]. However recent studies prove that Mebomian gland secretion (MGS) of many mammals (including humans, steers, etc.) does not contain polar lipids at all [6,7 and

\footnotetext{
* Corresponding author at: Department of Biochemistry, Faculty of Biology, University of Sofia “St. Kliment Ohridski”, 8 Dragan Tsankov Blvd., 1164 Sofia, Bulgaria. Tel.: +359 28167 204; fax: +35928656641.

E-mail address: ggeorg@biofac.uni-sofia.bg (G.As. Georgiev).
}

Refs. there in]. In addition some older [8] and some very recent investigations $[9,10]$ show that variety of polar lipids present in the tear's aqueous layer which therefore can serve as a potential source of polar lipid (PL) molecules for the air/tear interface. Thus currently unclear remain the origin of PLs, whether they present and in what amount in the surface lipid layer of tears, and what is their eventual role for TF functionality. PLs are interesting also from pharmaceutical point of view. At present they are used as compounds of artificial tears (Tears Again, Soothe, Refresh Dry Eye Therapy, FreshKote, Refresh Endura) targeted to replenish the TF lipid layer of patients with Dry Eye Syndrome. Although the number of lipid-containing artificial tears on the market still remains relatively limited, in vivo studies demonstrated that formulations containing phospholipids and other PLs significantly increase the non-invasive break-up time of TF in laboratory animals and humans [11-17].

Thus considering the pharmaceutical applications and the potential physiological role it is worth to study the surface interactions of Meibomian gland secretion with PLs. For the purpose we formed mixed pseudo-binary films of MGS and polar lipid, Egg Sphingomyelin (SM) or Dipalmitoylphosphatidylcholine (DPPC), at the 
air/water interface of Langmuir surface balance. Langmuir surface balance was already successfully employed in pioneer studies of the interactions between tear proteins and meibium [18-23]. SM and DPPC are chosen because they were used in previous studies as synthetic mimics of TF polar lipids [24,25]. DPPC is selected also due to its broad use in pharmaceutical formulations like the exogenous lung surfactant preparations aimed to achieve low equilibrium and dynamic surface tensions [26]. The behavior of the mixed films during slow quasi-equilibrium compression and during fast dynamic compression-decompression is registered by measurements of surface pressure and surface potential, and by monitoring film morphology with Brewster Angle Microscopy (BAM). Quasiequilibrium compression isotherms are used to calculate the excess Gibbs and Helmholtz energy of mixing between MGS and PLs and thus to evaluate the interactions between the lipid compounds at the interface. Deviations from ideal 2D mixing behavior have been interpreted as intermolecular attraction or repulsion in true two component monolayer. In the case considered here, with thicker lipid aggregates present in the surface film, they indicate more complex transitions associated with aggregation of lipid molecules in thick surface domains or with the domains disaggregation at the interface. The effects of PLs on the mixed film's elastic moduli of area compressibility, morphology and capability to attain high surface pressures are also examined.

\section{Materials and methods}

\subsection{Materials}

Dipalmitoylphosphatidylcholine, DPPC, and Egg Sphingomyelin, SM (containing primarily $\mathrm{N}$-linked palmitate) were purchased from Avanti Polar Lipids (Alabama, USA). $\mathrm{NaCl}$ was purchased from Merck (Germany).

Bovine Meibomian gland secretions were used in these experiments. The composition of bovine and human MGS is highly similar $[6,27]$. As it is easier to obtain sufficient amount of material of consistent composition, bovine MGS is the commonly used standard in studies of meibium surface properties [18,20,28]. MGS was extracted from bovine eyelids obtained from the abattoir by gently squeezing the lid borders as it was previously described $[18,20,28]$. The secretions were dissolved in chloroform, centrifuged and evaporated in a rotary concentrator at $25^{\circ} \mathrm{C}$ for $15 \mathrm{~min}$. Then samples were weighted, dissolved in chloroform at concentration $1 \mathrm{mg}$ $\mathrm{lipid} / \mathrm{ml}$ and stored at $-80^{\circ} \mathrm{C}$ until used. The composition of the samples was checked by thin layer chromatography (data not shown) using hexane/ether/acetic acid (80:20:2, v/v) as in [8] and revealed presence mainly of wax and sterol esters; phosphorylated PLs were not detected. For molecular weight of MGS we accepted $720 \mathrm{Da}$ as this value was used in previous works [18].

The saline solution $(0.15 \mathrm{M} \mathrm{NaCl})$ utilized as a subphase in Langmuir surface balance experiments was prepared with bidistilled water (Millipore) with conductivity less than $0.1 \mu \mathrm{S}$.

\subsection{Langmuir surface balance experiments}

A computer-controlled Langmuir type film balance ( $\mu$ Trough $\mathrm{XL}$; Kibron, Helsinki, Finland) equipped with a $\mu$ Trough XL (surface area $225 \mathrm{~cm}^{2}$, volume $40 \mathrm{ml}$ ) was used to simultaneously measure $\pi-A$ and surface potential-area $(\Delta V-A)$ isotherms, using the embedded features of the control software (FilmWare 3.57; Kibron). The indicated MGS/PL mixtures were dissolved in chloroform and spread over the air/saline solution interface with a Hamilton microsyringe. Then acrylic cover was put over the trough to protect the surface from dust contaminations and to suppress subphase evaporation. After 5 min equilibration (to ensure evapo- ration of the organic solvent), film area compression was started using two symmetrically moving barriers. Two types of measurements were performed: (i) slow quasi-equilibrium compression and (ii) fast dynamic compression-decompression cycling. In quasi-equilibrium compression experiments, the compression rate was $4 \AA^{2} /$ chain/min (considering the film compounds as lipids with two acyl chains) to allow for the reorientation and relaxation of the lipids in the film. The dynamic compression-decompression cycling was performed at the maximal possible compression rate at which there was no leakage of the film: $36 \AA^{2} / \mathrm{chain} / \mathrm{min}$. Surface pressure $(\pi)$ was monitored with a metal alloy probe hanging from a high precision microbalance (KBN 315; Kibron) connected to a computer and is defined as follows:

$\pi=\gamma_{0}-\gamma$

where $\gamma_{0}$ is the surface tension of the air/buffer interface and $\gamma$ is the value for surface tension in the presence of a lipid film compressed to varying packing densities. Thus as Eq. (1) shows, high surface pressure corresponds to low surface tension of the films. Monolayer surface potential $\Delta V$ was also registered during the quasi-equilibrium compressions using the vibrating plate method ( $\mu$ Spot; Kibron) as described by Brockman [29,30]. All isotherms were recorded at $28^{\circ} \mathrm{C}$ and were repeated at least three times to ensure reproducibility. The difference between the repetitions was up to two percents. The morphology of the films during compression was observed with MicroBAM2 (Nima Techology Ltd., UK).

\subsection{Analysis of the quasi-equilibrium compression isotherms}

We evaluated the intermolecular interaction in the pseudobinary lipid films, that consist of MGS as one compound and PL as second compound, at various surface pressures on the basis of the deviations of experimentally obtained mean molecular areas $\left(A_{\mathrm{m}}\right)$ from those of ideal mixtures $\left(A_{12}\right)$ :

$A_{12}=A_{1} X_{2}+A_{2} X_{2}$

where $A_{1}$ and $A_{2}$ are the molecular areas of pure components 1 and 2 , and $X_{1}$ and $X_{2}$ are molar fractions of components 1 and 2 , respectively. The value of $A_{12}$ corresponds to the mean molecular area in the mixture constituted of non-interactive or completely immiscible molecules. The negative deviation of $A_{\mathrm{m}}$ from $A_{12}\left(\triangle A=A_{\mathrm{m}}-A_{12}<0\right)$ indicates attractive interactions between the lipid molecules, while positive deviation of $A_{\mathrm{m}}$ from $A_{12}\left(\triangle A=A_{\mathrm{m}}-A_{12}>0\right)$-repulsive interactions between the lipid molecules.

In order to evaluate the miscibility of the lipids we calculated the excess Gibbs energy of mixing, $\Delta G$, as an integral of the deviation, $\Delta A$, over the surface pressure $\pi$ as given by Goodrich [31]:

$\Delta G=\int_{0}^{\pi}(\Delta A) d \pi=\int_{0}^{\pi}\left(A_{12}-X_{1} A_{1}-X_{2} A_{2}\right) d \pi$

Some lipid films are more sensitive to changes in the surface pressure than in the molecular area. Therefore it is convenient to analyze the excess property also by monitoring changes in the surface pressure at any given value of the molecular area. The ideal value of surface pressure of the mixed monolayer, $\pi_{A \text {,ideal }}$, at any given molecular area, $A$, can be determined from those of the pure monolayers at the same given area using the equation:

$\pi_{A, \text { ideal }}=X_{1}\left(\pi_{1}\right)_{A}+X_{2}\left(\pi_{2}\right)_{A}$

where $\left(\pi_{1}\right)_{A}$ and $\left(\pi_{2}\right)_{A}$ represent the surface pressures of the pure components at an area per molecule $A$. The interactions between the two components can be quantitatively studied by the surface pressure increment, $\Delta \pi$, which is calculated as the difference 

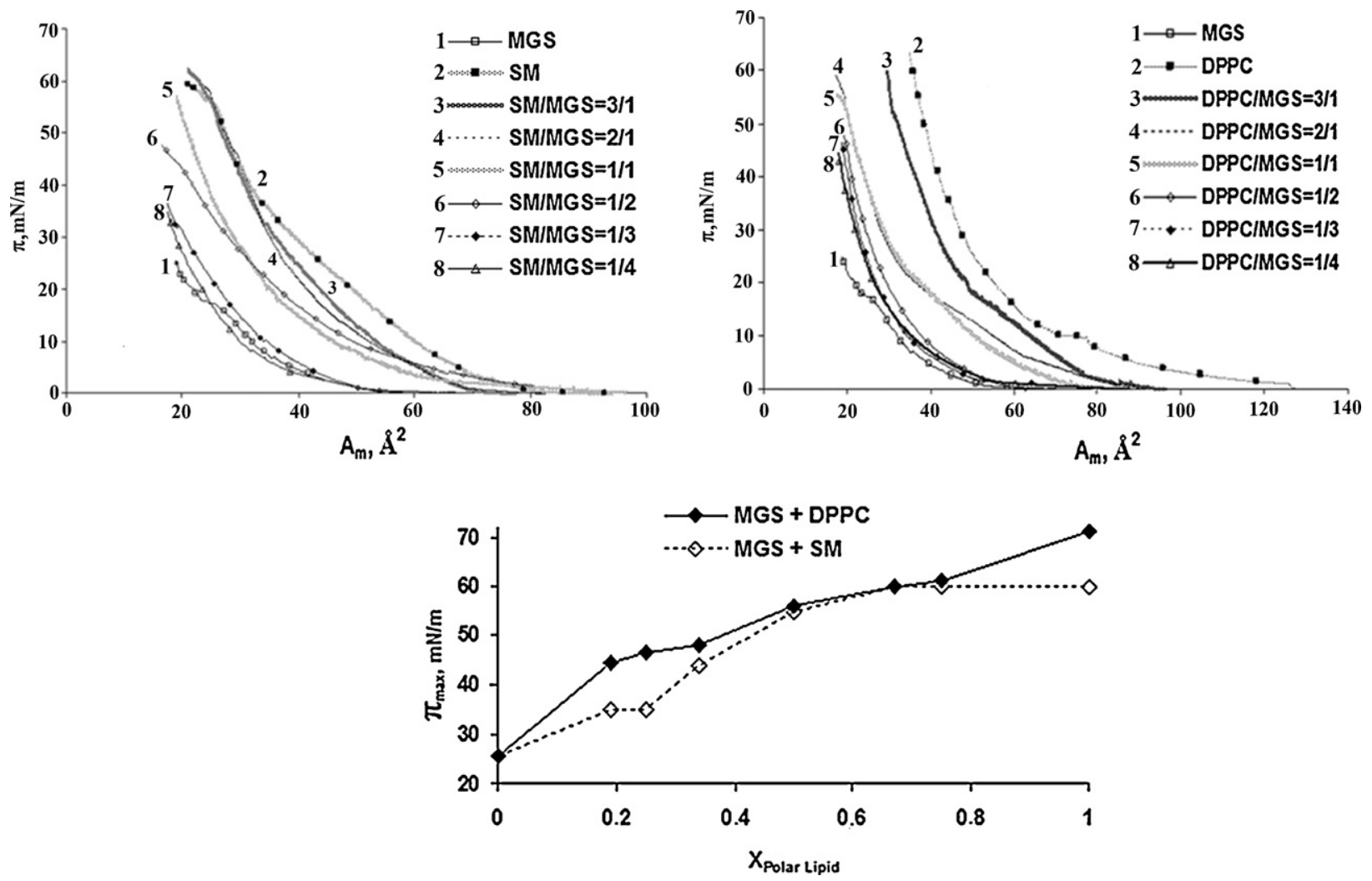

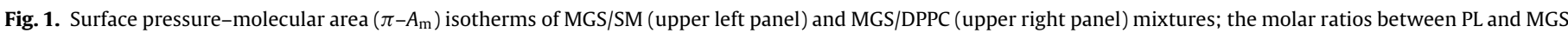

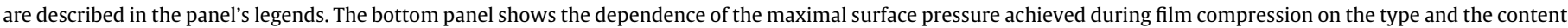
of the polar lipid.

between the ideal surface pressure, $\pi_{A \text {,ideal }}$, and the experimental value, $\pi_{A, \text { exptl }}$ :

$\Delta \pi=\pi_{A, \text { exptl }}-\pi_{A, \text { ideal }}$

Positive $\Delta \pi$ increments indicate a pressure increase effect, equivalent to an area expansion effect, and suggests a net repulsive interaction between the two components in the case of true two component monolayer. Negative surface pressure increments are indicative of area reduction effect, equivalent to an area contraction effect, which indicates a net attractive interaction between the two components in the case of true monolayer.

The surface pressure increments can be used to obtain the Helmholmtz excess energy of mixing, $\Delta A_{H}$, as it was first suggested by Gershfeld [32]. We calculated $\Delta A_{H}$ as described in [33] by the equation:

$\Delta A_{H}=\int_{A}^{A_{0}} \pi_{12} d A-x_{1} \int_{A}^{A_{0}} \pi_{1} d A-x_{2} \int_{A}^{A_{0}} \pi_{2} d A$

where $A_{0}$ is the area where $\pi$ gives the first increment from $\pi=0$ and $A$ is the molecular area at which the Helmholtz energy is to be calculated.

As in our case the surface films contained thicker multilayer lipid aggregates (i.e. films were not true monolayers), the surface pressure/surface area increments and the excess energies ( $\Delta G$ and $\left.\Delta A_{H}\right)$ calculated by Eqs. (2)-(6) did not measure 2D intermolecular attraction or repulsion. Here the quantities indicated more complex transitions related to (i) aggregation of lipid molecules in thick surface domains or to (ii) the domains disaggregation at the interface.

Film isothermal compressibility at a given surface pressure $\pi$ was calculated from surface pressure-molecular area $(\pi-A)$ data using:

$C_{S}=-\frac{1}{A_{\pi}}\left(\frac{d A}{d \pi}\right)_{T}$

where $A \pi$ is the area per molecule at the indicated surface pressure $\pi$. Further we plotted the dependence of elastic moduli of area compressibility (also known as reciprocal isothermal compressibility), $C_{s}^{-1}$, on $\pi$ as in [34]. In case of true monolayer it is possible to identify the physical state of the film at given surface pressure using the criteria of Davies and Rideal [35]: $C_{s}^{-1}<100 \mathrm{mN} / \mathrm{m}$ corresponds to liquid-expanded state, $C_{s}^{-1}$ within $100-250 \mathrm{mN} / \mathrm{m}$ to liquid-condensed state and $C_{s}^{-1}>250 \mathrm{mN} / \mathrm{m}$ to solid state of the film.

\section{Results}

\subsection{Quasi-equilibrium compression of mixed films of MGS and PLS}

The $\pi-A$ isotherms of MGS, SM, DPPC and the pseudo-binary MGS/PL mixtures are presented in Fig. 1. The isotherms of the three "pure" compounds, MGS, SM and DPPC coincide well with the previously published results obtained under similar conditions [18-20,29,34]. MGS isotherm shows low slope (i.e. high compressibility) with characteristic inflexion at $15-17 \mathrm{mN}$ and maximal surface pressure of $25 \mathrm{mN} / \mathrm{m}$ as reported in [18-20].

It can be seen that the increase of the molar fraction of PL in the MGS/PL mixed films results in the increase of the lift-off area (the area per molecule where $\pi$ gives the first increment from $\pi=0$ ) and of the value of the maximal surface pressure achieved at the end of film compression (Fig. 1 lower panel). The addition of 0.2 molar parts PL increases $\pi_{\max }$ from $25 \mathrm{mN} / \mathrm{m}$ up to $35 \mathrm{mN} / \mathrm{m}$ and $45 \mathrm{mN} / \mathrm{m}$ in the presence of SM and DPPC, respectively. The effect of 

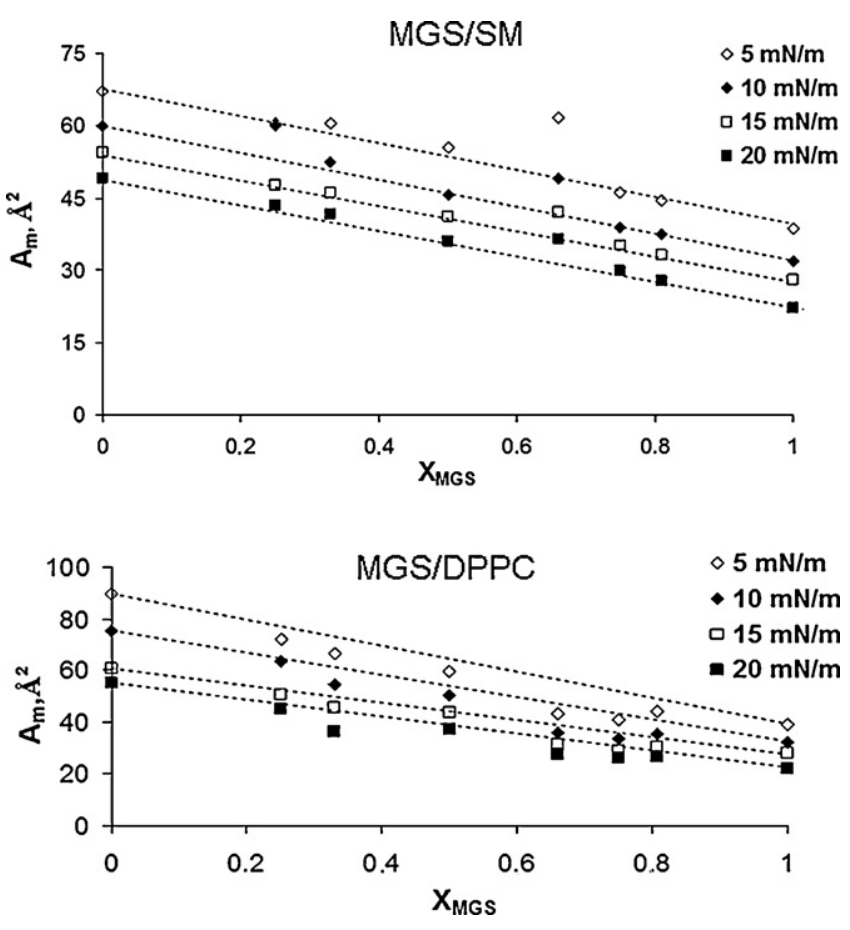

Fig. 2. Dependence of mean molecular area $\left(A_{\mathrm{m}}\right)$ on composition of MGS/SM (upper panel) and MGS/DPPC (lower panel) films at surface pressures of 5, 10, 15 and $20 \mathrm{mN} / \mathrm{m} ; X_{\text {MGs }}$ denotes the molar part of Meibomian lipids in the pseudo-binary films. The dashed lines show the trends of $A_{\mathrm{m}}-X_{\mathrm{MGS}}$ dependences as calculated by the additivity rule (Eq. (2)) at the given surface pressures.

DPPC on $\pi_{\max }$ is higher than the effect of SM in the pseudo-binary films up to $X_{\mathrm{MGS}}=0.5$, while for lower meibium contents the effect of the two PLs equalizes.

To analyze the interaction between MGS and PL molecules we plot the mean molecular area as a function of the molar fraction of meibium at surface pressures of $5,10,15$ and $20 \mathrm{mN} / \mathrm{m}$ (Fig. 2). The shape of the dependences of the molecular area on film composition is similar for all selected $\pi$.

In case of MGS/SM films for most of the compositions, the experimentally measured mean molecular areas coincide well with the theoretically calculated, according to the additivity rule (Eq. (2)), values. Only at $X_{\mathrm{MGS}}=0.63$ area expansion effect is observed, i.e. the experimental mean molecular area is significantly higher than the theoretically expected one. In contrast to MGS/SM mixtures, the experimentally determined mean molecular areas in MGS/DPPC films are lower than the values expected in case of area additivity for the entire range of MGS/SM ratios. The peak values of this area contraction effect are realized at $X_{\mathrm{MGS}}=0.33$ and 0.66 where the experimentally determined molecular areas are about $10-15 \AA^{2}$ smaller than the calculated ones.

Further the data in Fig. 2 are used to calculate by Eq. (3) the Gibbs excess energy of mixing between MGS and PLs at the four chosen surface pressures (Fig. 3).

For most of compositions of MGS/SM films (Fig. 3 upper panel), the value of $\Delta G$ is zero or just slightly deviates $(<0.2 \mathrm{~kJ} / \mathrm{mol}$ for the majority of the points) to positive values which indicates from no interaction to weak interaction between the lipid molecules as it is already suggested from the relatively weak positive deviation of the mean molecular area from additivity. As in the $A_{\mathrm{m}}-X_{\mathrm{MGS}}$ dependence (Fig. 2 upper panel) the peaks of the positive $\Delta G$ values are located at $X_{\mathrm{MGS}}=0.3(\Delta G=0.45 \mathrm{~kJ} / \mathrm{mol}$ at $\pi=10 \mathrm{mN} / \mathrm{m})$ and $0.6(\Delta G=0.63 \mathrm{~kJ} / \mathrm{mol}$ at $\pi=10 \mathrm{mN} / \mathrm{m})$. Concerning MGS/DPPC films, $\Delta G$ takes negative values for the entire compositional range. The magnitude of the negative excess energy generally increases with increasing surface pressure and strongest interactions are
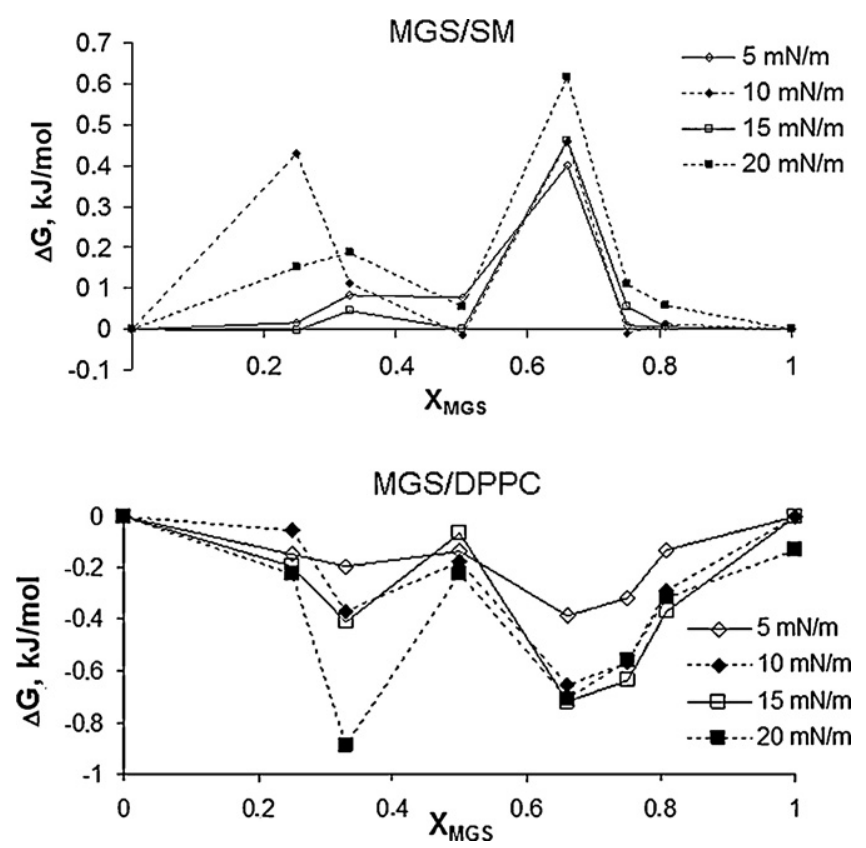

Fig. 3. Dependence of Gibbs excess energy $(\Delta G)$ of mixing on composition of MGS/SM (upper panel) and MGS/DPPC (lower panel) films at surface pressures of $5,10,15$ and $20 \mathrm{mN} / \mathrm{m} ; X_{\mathrm{MGS}}$ denotes the molar part of Meibomian lipids in the pseudo-binary films.

observed at $X_{\mathrm{MGS}}=0.35(\Delta G=-0.9 \mathrm{~kJ} / \mathrm{mol}$ at $\pi=20 \mathrm{mN} / \mathrm{m})$ and $X_{\mathrm{MGS}}=0.66-0.75$ ( $\Delta G$ ranges from -0.6 to $-0.7 \mathrm{~kJ} / \mathrm{mol}$ at surface pressures of $10,15,20 \mathrm{mN} / \mathrm{m})$.

As the sensitivity of lipid films to changes in surface area and surface pressure might differ $[32,33]$ we analyzed the interactions of MGS with polar lipids also in terms of surface pressure increments calculated by Eq. (5) at three areas per molecule: 40,45 and $50 \AA^{2}$ (Fig. 4).

For MGS/SM films in the range of $X_{\mathrm{MGS}}$ from 0 to 0.66 the surface pressure increment shows positive values at $X_{\mathrm{MGS}}$ of 0.33 and 0.66 at $40 \AA^{2}$ per molecule. For the rest of compositions and for the rest of areas per molecule the value of $\Delta \pi$ is low and near to zero, which means that no significant interaction is realized of MGS and SM. When molar fraction of MGS is increased above 0.63 , negative surface pressure increment of $-6 \mathrm{mN} / \mathrm{m}$ at all areas per molecule is observed at $X_{\mathrm{MGS}}=0.7$.

MGS/DPPC films for all compositions show negative surface pressure increments that increase when the molecular area is lowered. Maximal negative $\Delta \pi$ increments (reaching up to $-14 \mathrm{mN} / \mathrm{m}$ ) are observed at $X_{\mathrm{MGS}}=0.33$ and 0.63 .

On the base of $\Delta \pi-X_{\mathrm{MGS}}$ by Eq. (6) the Helmholtz excess energy is calculated for the pseudo-binary mixtures (Fig. 5).

When the MGS molar fraction is increased to 0.66 in the MGS/SM films, $\Delta A_{H}$ values are positive at $40 \AA^{2}$ per molecule. The peaks are located at $X_{\mathrm{MGS}}$ of 0.33 and 0.66 per molecule, but as it can be seen even at these points the excess energy is low $(<0.4 \mathrm{~kJ} / \mathrm{mol})$. At the other $A_{\mathrm{m}}$ the excess energy of mixing is near to zero, i.e. there is no significant interaction. When meibium content is further increased $\left(X_{\mathrm{MGS}}>0.66\right)$ negative $\Delta A_{H}$ reaches up to $-0.4 \mathrm{~kJ} / \mathrm{mol}$ at $X_{\mathrm{MGS}}=0.7$.

Over the whole range of compositions MGS/DPPC mixtures displayed negative Helmholtz excess energy, whose magnitude increases with the decrease of the molecular area. Maximal negative $\Delta A_{H}$ (of up to $-1.8 \mathrm{~kJ} / \mathrm{mol}$ ) is observed at $X_{\mathrm{MGS}}=0.33$ and 0.66 .

For more complete characterization of the pseudo-binary film's structuredness and of the degree of orientation of molecular dipole moments in them, we measure the dependence of mono- 

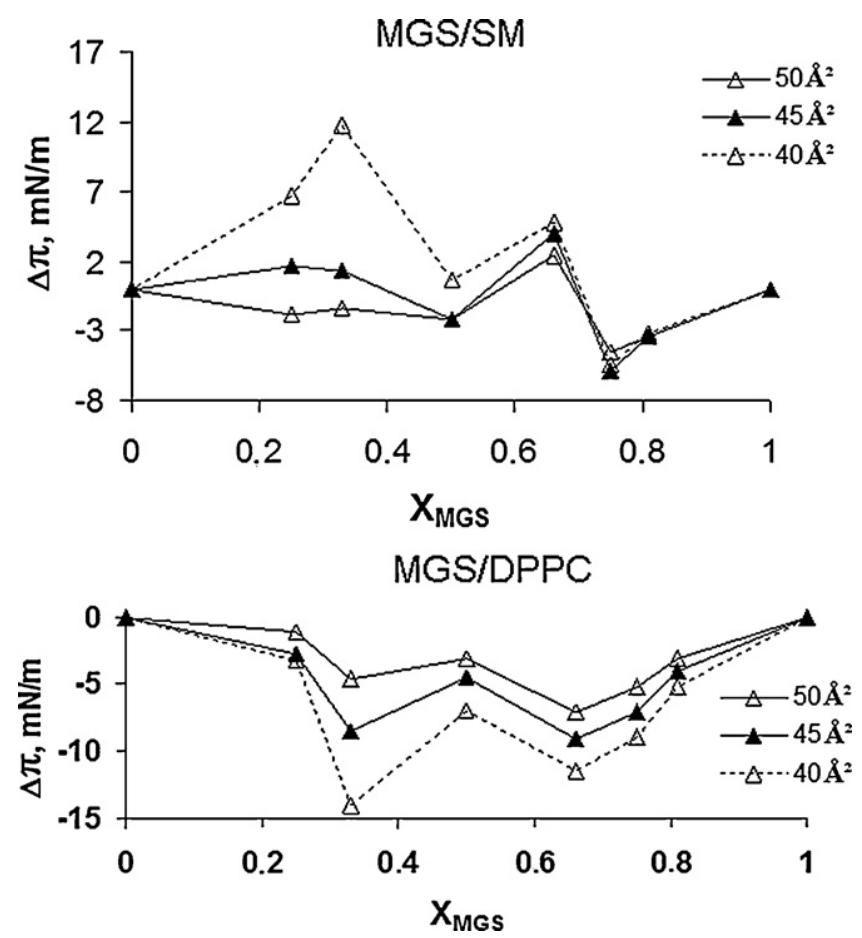

Fig. 4. Dependence of surface pressure increments $(\Delta \pi)$ on composition of MGS/SM (upper panel) and MGS/DPPC (lower panel) films at 40, 45 and $50 \AA^{2}$ per molecule; $X_{\text {MGS }}$ denotes the molar part of Meibomian lipids in the pseudo-binary films.

layer surface potential on molecular area during compression (Fig. 6).

$\Delta V-A$ isotherms of all MGS/PL mixtures show similar shapes, but the surface potential of the MGS/DPPC films are shifted to higher values in comparison to MGS/SM films. We further use $\Delta V-A$ isotherms (Fig. 6) in order to evaluate how the alteration of the ratio between polar lipid and meibium reflects on the films surface potential at fixed surface pressures, i.e. at constant molecular packing at the interface $[29,36]$. For the purpose the dependence of $\Delta V$
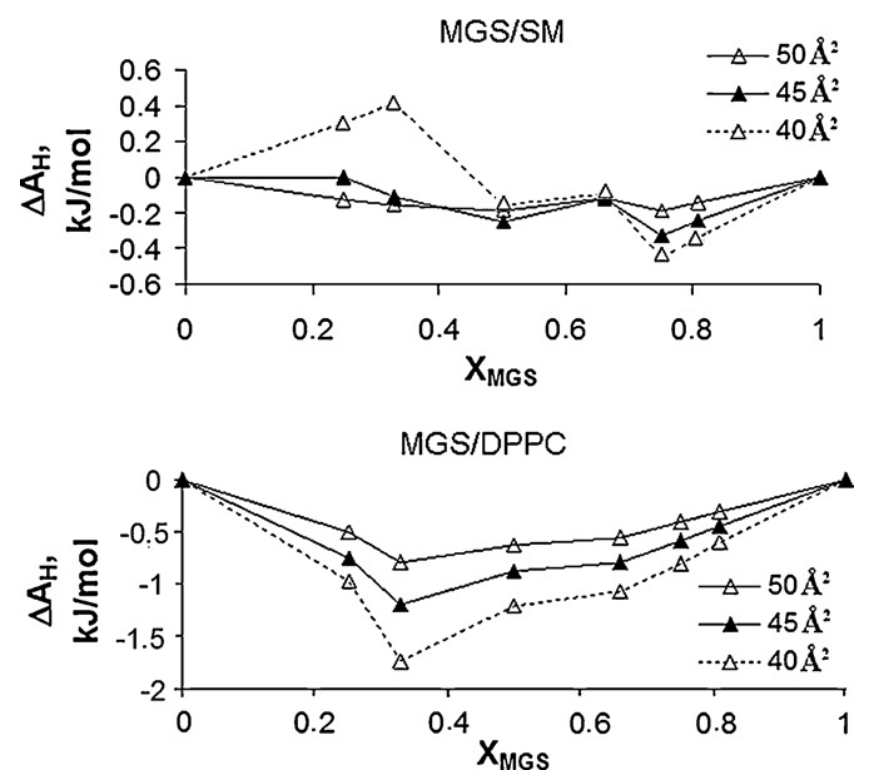

Fig. 5. Dependence of Helmholtz excess energy $\left(\Delta A_{H}\right)$ of mixing on composition of MGS/SM (upper panel) and MGS/DPPC (lower panel) films at 40, 45 and $50 \AA^{2}$ per molecule; $X_{\mathrm{MGS}}$ denotes the molar part of Meibomian lipids in the pseudo-binary films.

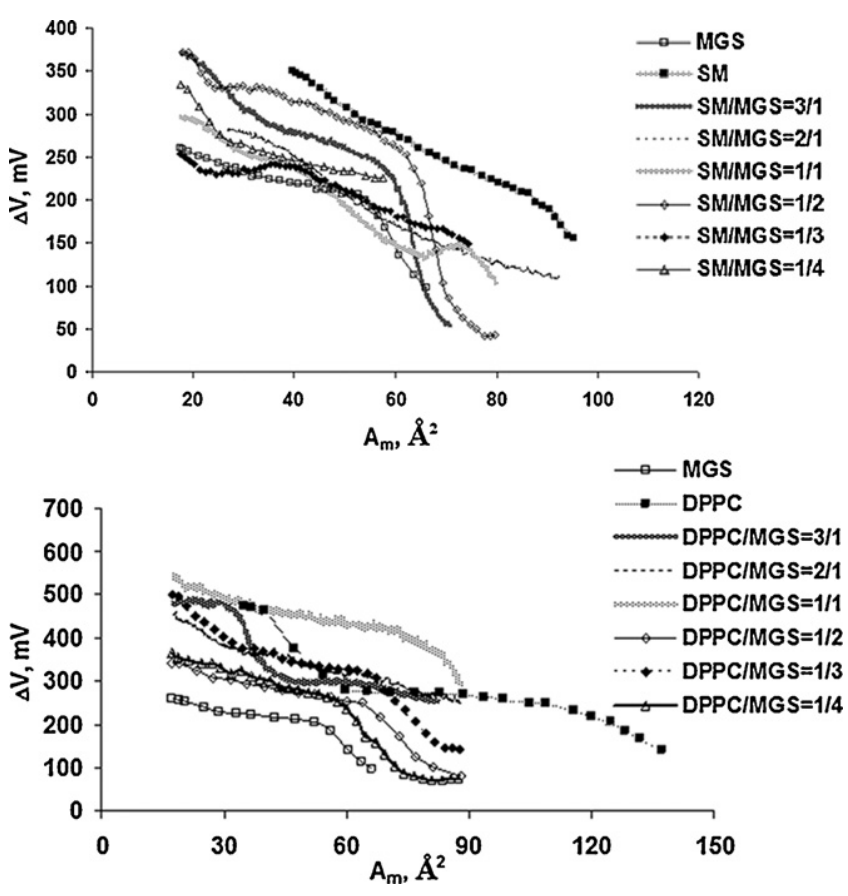

Fig. 6. Surface potential-molecular area $\left(\Delta V-A_{m}\right)$ isotherms of MGS/SM (upper panel) and MGS/DPPC (lower panel) mixtures.

on $X_{\text {MGS }}$ (Fig. 7) for the studied lipid mixtures is plotted at $\pi=5,10$, 15 and $20 \mathrm{mN} / \mathrm{m}$.

For films containing one and the same PL (SM or DPPC) the trends of the surface potential dependencies on the film composition are identical for all surface pressures, with $\Delta V-X_{\text {MGS }}$ curves shifting to slightly higher voltages with the increase of $\pi$. However the shape of $\Delta V-X_{\mathrm{MGS}}$ dependencies for the two lipid mixtures (ones that contain SM, and others - DPPC) significantly differs. For MGS/SM mixtures $\Delta V$ passes through minimums: at $X_{\mathrm{MGS}}=0.33$ and at $X_{\mathrm{MGS}}=0.75$. In case of MGS/DPPC films for $X_{\mathrm{MGS}}$ range from 0.25 to 0.82 molar parts, the values of the surface potential of the pseudo-binary films are higher than the surface potential of the
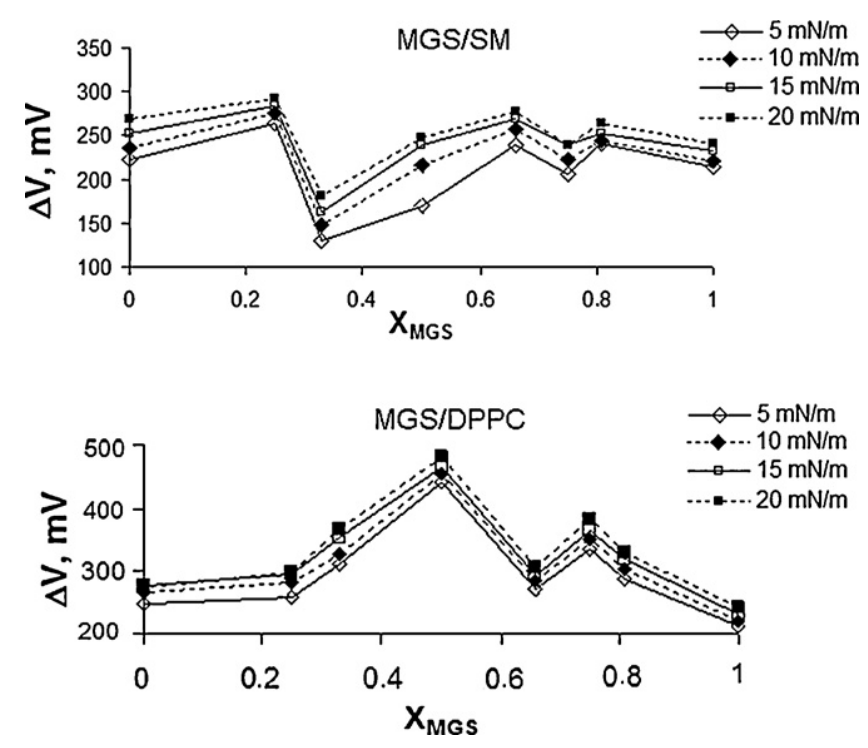

Fig. 7. Dependence of surface potential $(\Delta V)$ on composition of MGS/SM (upper panel) and MGS/DPPC (lower panel) films; $X_{\text {MGS }}$ denotes the molar part of Meibomian lipids in the pseudo-binary films. 

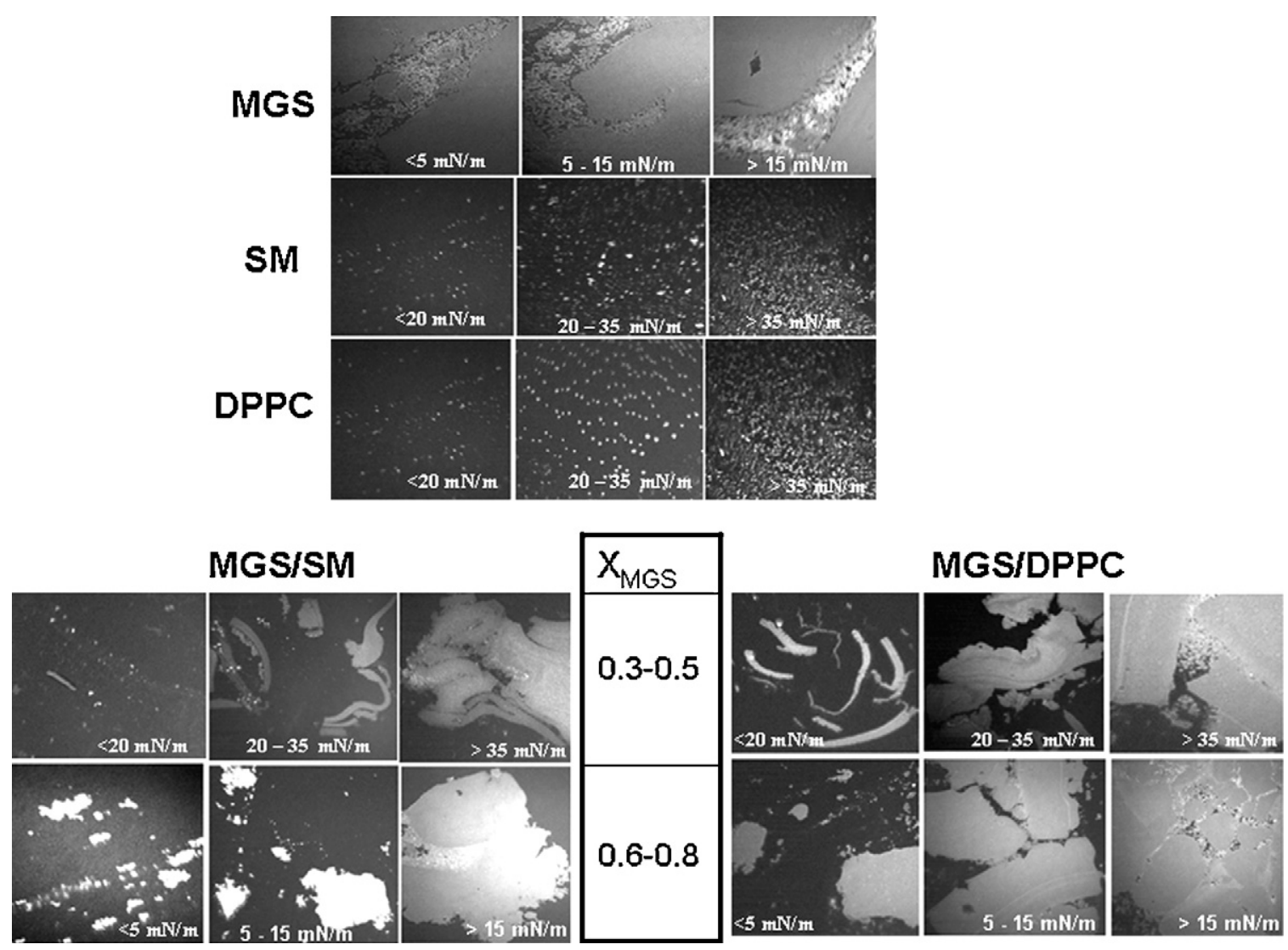

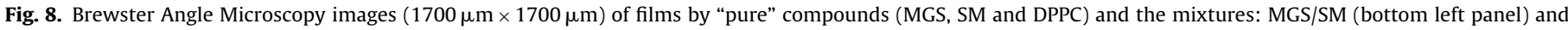

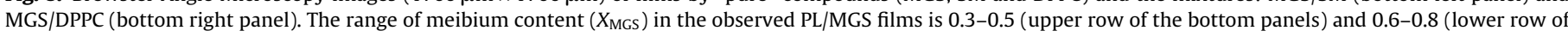
the bottom panels).

pure compounds (MGS and DPPC). There are two peaks of $\Delta V$ : at 0.5 and 0.82 molar parts meibium.

The changes in the morphology of the MGS/PL films when varying the ratio between the compounds are monitored with BAM (Fig. 8).

Pure MGS forms bright thick aggregates with condensed-like appearance even at low surface pressures $(<5 \mathrm{mN} / \mathrm{m})$ which contain darker stripes of thinner regions. When the surface pressure is increased these darker stripes tend to close and the bright meibium islands get more uniform and homogeneous. The two polar lipids does not display significant amount of domains at $\pi<20 \mathrm{mN} / \mathrm{m}$ within the separation of the microBAM which agrees well with the observations in [33]. At higher $\pi$ both PL display circular domains that gradually increases in number during compression. When SM is in equimolar content or in excess to MGS $\left(X_{\mathrm{MGS}}=0.33-0.5\right)$, the domains are observed in the pseudo-binary films only at high surface pressures. These aggregates morphologically resemble the meibium islands but are in much lower amount when compared to pure MGS. When the content of meibium is increased $\left(X_{\mathrm{MGS}}=0.66-0.8\right)$ thick lipid islands start to form more readily at surface pressures equivalent to the ones at which the aggregates appear in pure MGS films.

When DPPC is present at high concentrations in the pseudobinary films $\left(X_{\mathrm{MGS}}=0.33-0.5\right)$ the formation of meibium-like islands shifts to higher surface pressures when compared to MGS. The size of the lipid aggregates in these DPPC/MGS films resemble pure meibium and their quantity is much higher in comparison to SM-containing mixtures with similar PL amount. When the content of meibium in the films is elevated $\left(X_{\mathrm{MGS}}=0.66-0.8\right)$, the size and the quantity of the domains and the surface pressures at which they appear are equivalent to the ones for pure MGS.
Further we studied the effect of PLs on the elasticity modulus of area compressibility of the mixed films. The surface pressure $C_{s}^{-1}$ dependencies are presented in Fig. 9.

It can be seen that $\pi-C_{S}^{-1}$ trends of pure MGS displays reversal of the slope between 15 and $20 \mathrm{mN} / \mathrm{m}$ which indicates phase transition taking place in the film plane [34]. It corresponds to the change in the slope of the meibium $\pi-A_{\mathrm{m}}$ isotherm at these surface pressures. BAM observations demonstrated that in that $\pi$ range, the number and the size of the dark black stripes inside the MGS islands tend to diminish, while the bright condensate grows and gets more homogeneous. Reversal of the slope shows also the $\pi-C_{s}^{-1}$ curve of DPPC between 10 and $12 \mathrm{mN} / \mathrm{m}$. At the same surface pressures is localized the plateau like region of the Dipalmitoylphosphatidylcholine $\pi-A_{\mathrm{m}}$ isotherm where the liquidextended to liquid-condensed phase transition is known to occur [34]. For SM the dependence of the reciprocal isothermal compressibility on $\pi$ shows broad and not clearly pronounced change in the slope between 20 and $38 \mathrm{mN} / \mathrm{m}$ which corresponds to the slight change in the slopes of the SM compression isotherm at these surface pressures. It can be also seen that in agreement with [34] at lower $\pi(<20 \mathrm{mN} / \mathrm{m})$ the reciprocal compressibility of SM monolayers exceeds the one of DPPC films, while when surface pressure is increased $C_{S}^{-1}$ of DPPC monolayers is higher. When MGS and PLs are mixed $\pi-C_{s}^{-1}$ trends get smoother and do not display distinguished regions with reversed slopes. This indicates that during the compression of the pseudo-binary films there are no clearly pronounced phase transitions taking place. That correlates with the film morphologies registered by BAM where lipid condensates are monitored in a very broad $\pi$ range and there is no threshold surface pressure after which the domain formation is triggered. 

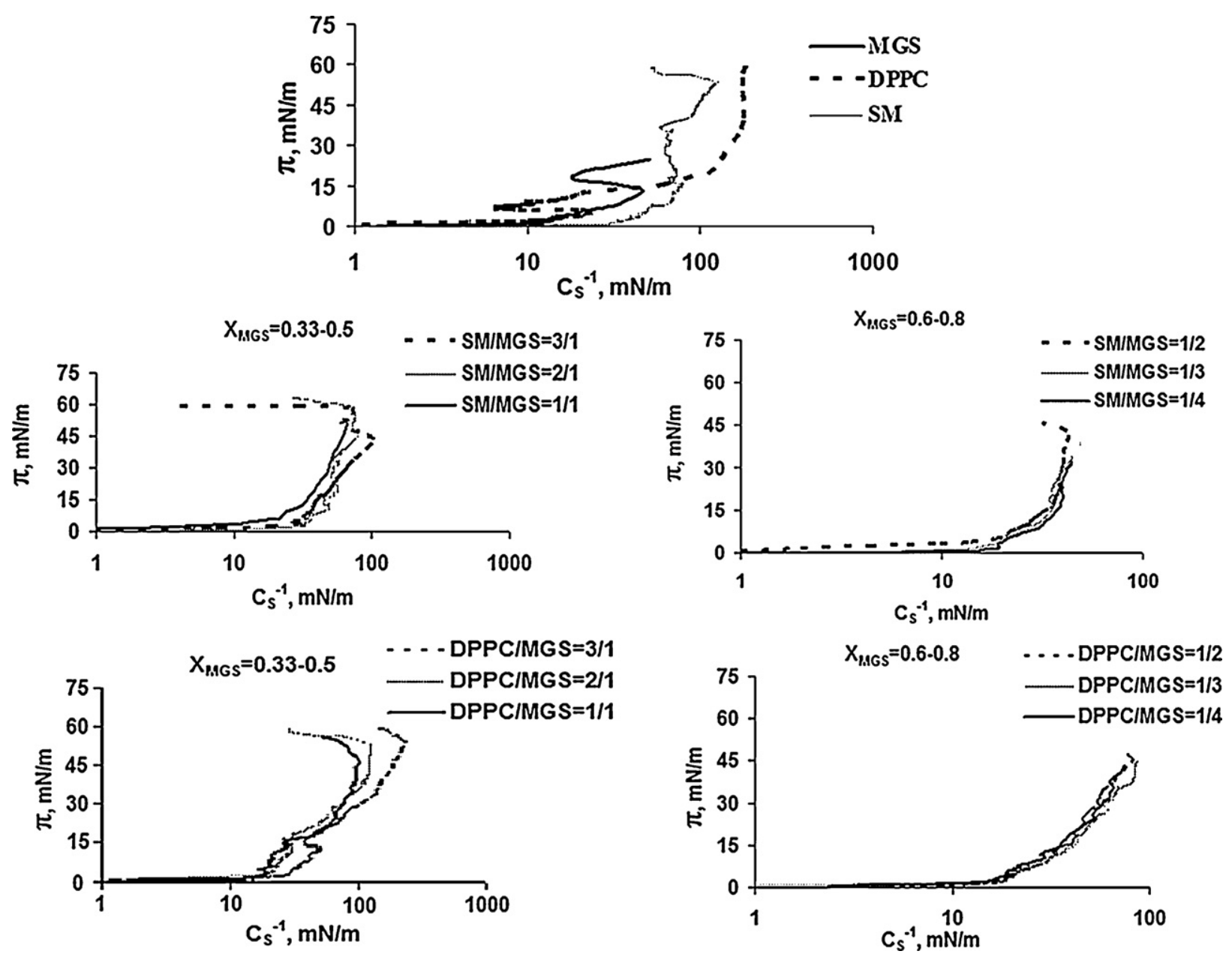

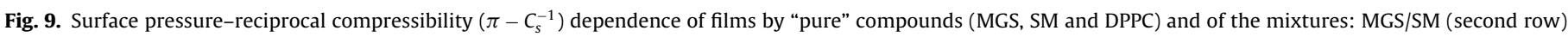
and MGS/DPPC (third row). The ratio between PL and MGS is denoted in the panel's legends.

Table 1 summarizes the data concerning the maximal elasticity of the examined compositions.

The maximal reciprocal compressibility of MGS is $47 \mathrm{mN} / \mathrm{m}$. It can be seen that both PL display $C_{s}^{-1}>100 \mathrm{mN} / \mathrm{m}$, i.e. achieve liquid-condensed phase during compression, with the elasticity of DPPC $(214 \mathrm{mN} / \mathrm{m})$ being significantly higher than the elasticity of SM $(110 \mathrm{mN} / \mathrm{m})$. The addition of PL to MGS solidifies the surface films and increases $C_{s}^{-1}$. Still the effect of DPPC is much stronger than the one of SM for all MGS/PL ratios. The measured reciprocal compressibility of pure SM and DPPC is somewhat lower than the previously reported [34]. One possible reason is that our experiments are performed not at $23-24^{\circ} \mathrm{C}$ as in other works [34,37] but at $28-29^{\circ} \mathrm{C}$ which might result in formation of more fluid films. Our choice is motivated by the data that corneal temperature is about $32-33^{\circ} \mathrm{C}[38,39]$. Thus concerning the heat exchange between the ocular surface and the environment, tear temperature of $28-30^{\circ} \mathrm{C}$ seems reasonable at temperate climate. Also we use compression rate of $4 \AA^{2} /$ chain/min as it was done in [29], while in $[34,37]$ even slower compression rates are implied. We found that at slower compression, the evaporation artefacts become significant and hard to overcome at the temperature at which the experiments are performed.

The studied films are subjected to dynamic isocycling, i.e. rapid and continuous compression/decompression cycling until constant in time surface pressure-area loop is obtained. Twenty cycles are performed with each composition and normally constant $\pi-A$ hys-

Table 1

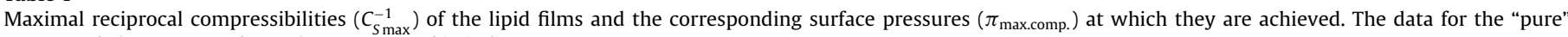
compounds (MGS, SM and DPPC) are presented in italic.

\begin{tabular}{|c|c|c|c|c|}
\hline \multirow[t]{2}{*}{ Film composition } & \multicolumn{2}{|c|}{$C_{S \max }^{-1}(\mathrm{mN} / \mathrm{m})$} & \multicolumn{2}{|c|}{$\pi_{\text {max.comp. }}(\mathrm{mN} / \mathrm{m})$} \\
\hline & MGS/SM & MGS/DPPC & MGS/SM & MGS/DPPC \\
\hline MGS & & & & \\
\hline$S M$ & & & & \\
\hline$D P P C$ & & & & \\
\hline $\mathrm{MGS} / \mathrm{PL}=1 / 3$ & 103 & 214 & 43 & 54 \\
\hline $\mathrm{MGS} / \mathrm{PL}=1 / 2$ & 93 & 121 & 44 & 45 \\
\hline $\mathrm{MGS} / \mathrm{PL}=1 / 1$ & 70 & 90 & 58 & 45 \\
\hline $\mathrm{MGS} / \mathrm{PL}=2 / 1$ & 45 & 67 & 43 & 49 \\
\hline $\mathrm{MGS} / \mathrm{PL}=3 / 1$ & 50 & 69.4 & 34 & 43 \\
\hline $\mathrm{MGS} / \mathrm{PL}=4 / 1$ & 52 & 67 & 35 & 36 \\
\hline
\end{tabular}



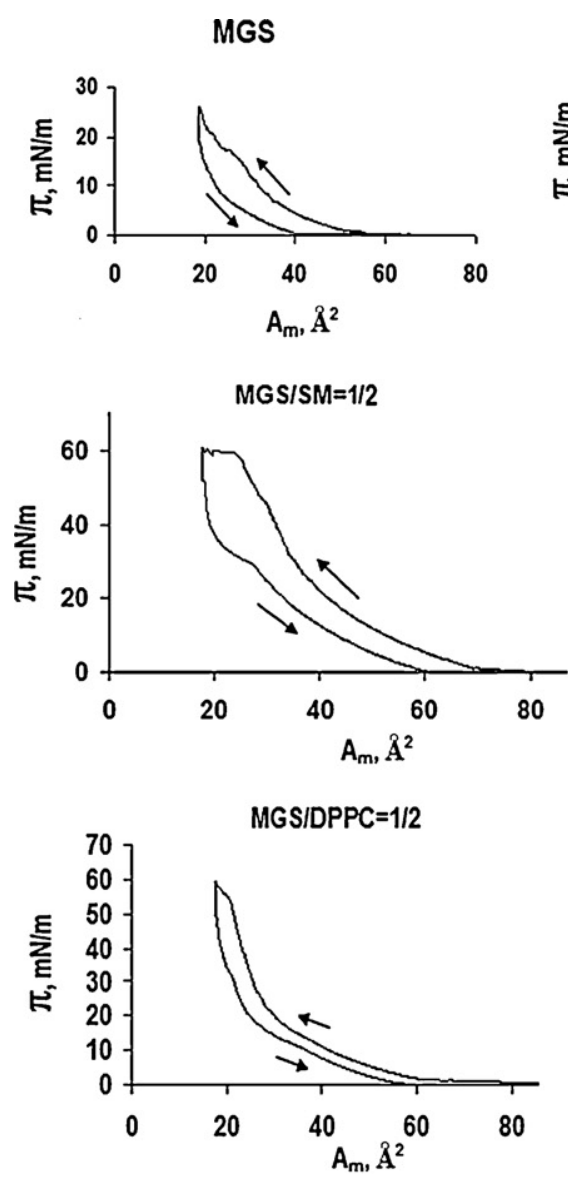
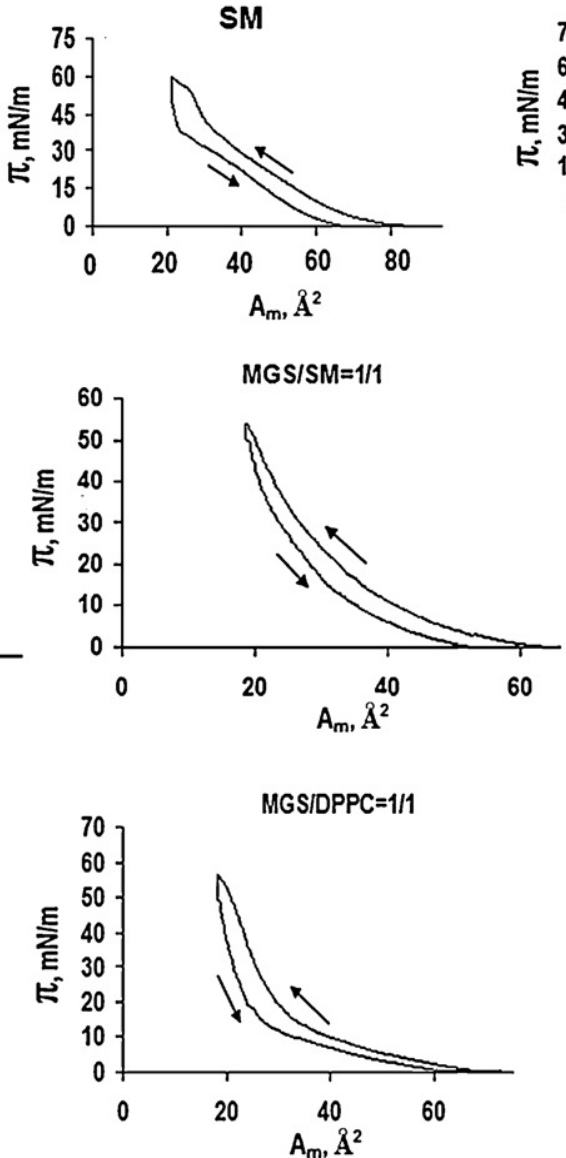

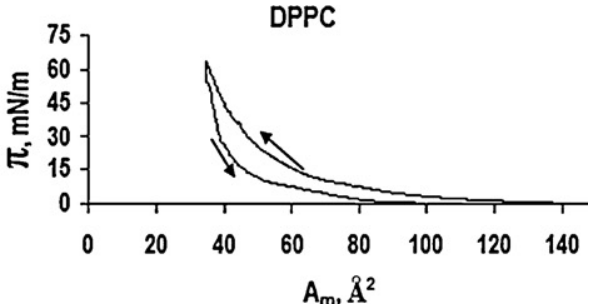

MGSISM=2/1

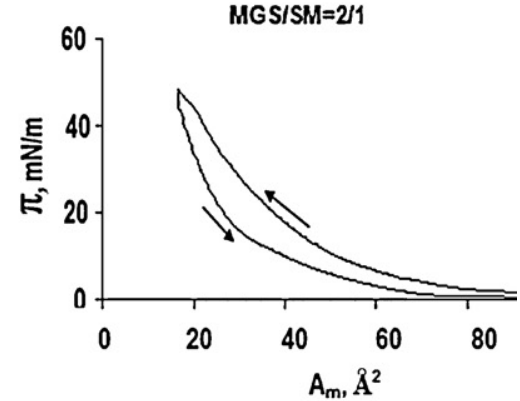

MGS/DPPC $=2 / 1$

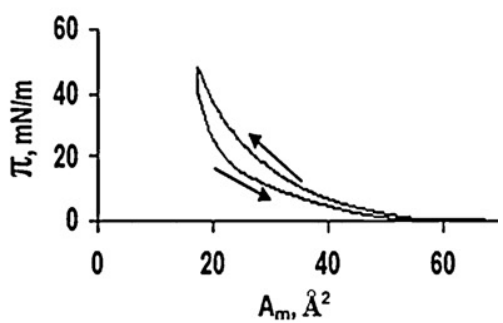

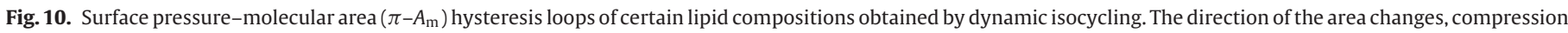
or decompression, is designated by arrows.

tersis is obtained between the third and the fifth cycles. The results for some films are shown in Fig. 10.

The dynamic isocycling does not change significantly the shape of the $\pi-A$ isotherms of the studied films when compared to the quasi-equilibrium compressions. All compositions show relatively low hysteresis, which is especially clearly seen for DPPC/MGS films.

\section{Discussion}

Polar lipids interact with Meibomian gland secretion in different manner and with different strength.

Prior to discuss this issue in more details it is worth to consider in what state MGS exists at the air/water interface. BAM images demonstrate that even at low surface pressures meibium forms large bright thick aggregates with some thinner black stripes amongst them. As $\pi$ increases these thick lipid islands tend to grow and merge and to fulfil the field of view while the black stripes almost disappear. Similar images are already observed with fluorescence microscopy by Millar et al. [20] in surface balance studies of MGS, and interpreted as lipid islands with some water channels in it, that gradually close during the film compression. The size of the aggregates and the intensity of the BAM images indicate that MGS actually does not exist as a monolayer even at zero surface pressure but instead forms thicker multilayer structures. Similar observations are also reported by others $[25,40]$. The property of meibium to form multilayers but not monolayers is easily explained in terms of MGS composition. The major molecules that comprise more than $90 \%$ of MGS are extremely hydrophobic (long chain wax and sterol esters, triglycerides, etc.) and spontaneously form thick lipid matrix bridged to the air/water interface by a layer of still unidentified more polar molecules [4,5,7].

Major consequence of that surface behavior is that the interactions between MGS and PLs cannot be interpreted as twodimensional one, but represent much more complex case of interaction between polar lipids and 3D meibium islands. Thus the measured Gibbs and Helmoltz excess energies do not indicate 2D attraction or repulsion, but actually provide quantitative measure about more sophisticated interfacial transitions, being: (i) aggregation of lipid molecules in thick domains when "negative" excess energies are calculated and (ii) disaggregation of the thick meibium islands-when "positive" excess energies are detected.

For most of the tested compositions SM shows practically no specific interaction with MGS, and the experimentally determined values of area and surface pressure increments are close to the predictions of the additivity rule (Eqs. (2) and (4)). Only when SM is in excess to MGS $\left(X_{\mathrm{MGS}}<0.5\right)$ weak interaction is measured by positive $\Delta A$ and $\Delta \pi$ increments and by positive but low $(<0.5 \mathrm{~kJ} / \mathrm{mol})$ excess energies. As demonstrated by BAM in that compositional range the amount of the meibium islands in the mixed films is decreased which suggests that domain disaggregation takes place. Significant amount of aggregates is observed only at high surface pressures.

In contrast to SM the other polar lipid, Dipalmitoylphosphatidylcholine, strongly interacts with meibium. Practically for the entire range of DPPC/MGS ratios, area contraction and negative surface pressure increments are observed, with the peak values located at the compositions with prevalence of DPPC $\left(X_{\mathrm{MGS}}<0.5\right)$. When the Gibbs and Helmholtz excess energies are calculated high negative values of up to $-1.8 \mathrm{~kJ} / \mathrm{mol}$ are obtained. The negative excess energies and the significant amount of meibium "islands" monitored 
by BAM over wide compositional range in the pseudo-binary films indicate that aggregation of the lipid molecules in thick domains takes place.

The difference in the mode and strength of interaction between the polar lipids and MGS is confirmed also by the dependencies of the surface potential on PL/MGS ratio. It is well known that $\Delta V$ is proportional to the capability of lipid headgroups to epitaxially structure water molecules and to the degree of normal orientation of the molecular dipole moments [29,30]. It might be expected that in multilayer films, where the lipid headgroup/water interface is efficiently screened by the lipid matrix, the molecular dipole orientation will be major determinant of the surface potential value. Thus the trends shown in Fig. 7 provide insight into how the order of the films changes when the lipid composition is varied. For MGS/SM mixtures $\Delta V$ passes through minimum at $X_{\mathrm{MGS}}=0.33$ and for a range of compositions $\left(X_{\mathrm{MGS}}=0.3-0.5\right)$ the surface potentials of the mixed films are lower than the values for the pure compounds. The result confirms decreased order of the films in excess of SM as suggested by the calculations of $\Delta G$ and $\Delta A_{H}$ and by the observation of films with BAM. Quite problematic is to evaluate what happens in SM/MGS films, when meibium is in excess $\left(X_{\mathrm{MGS}}=0.75\right)$. In this region the values obtained for $\Delta G$ are slightly positive, while $\Delta A_{H}$ is slightly negative. $\Delta V$ also shows weak drop at that point, but still the surface potentials are not lower than for pure SM and pure MGS. Concerning the low values of both excess energies and that BAM images indicate recovered formation of large meibium islands at the same surface pressures as in pure MGS films, probably the behavior of the system in MGS prevalence is close to ideal mixing.

For DPPC/MGS mixtures BAM images and the quantities describing the interaction, $\Delta A_{H}$ contraction (and subsequent $\Delta G$ calculation) and negative $\Delta \pi$ increments (with evaluation of $\Delta A_{H}$ ), pointed on significant affinity between DPPC and MGS resulting in their aggregation in thick domains in the surface layers. The picture is synonymously confirmed by the peaks in the surface potential trend and by the increased $\Delta V$ values of the pseudo-binary films for $X_{\mathrm{MGS}}=0.25-0.82$ when compared to the pure compounds (MGS and DPPC).

It is very hard to give definitive interpretation of the results. This is due to the very complex composition of meibium: an intricate puzzle of more than 300000 molecular species [9] currently subjected to intensive studies $[7,10]$. In addition the chemical "communication" of PLs with the thicker meibium islands might easily include not only 2D effects in the plane of the air/water interface, but also interactions between the lipid acyl chains and the non-polar molecules (wax and sterol esters, triglycerides, etc.) that reside in the hydrophobic matrix of the MGS aggregates. Back in 1997, McCulley and Shine [4,5] proposed a model based on the assumption that phospho- and sphingolipids represent up to $10 \%$ of meibium $[41,42]$. Now it is known that these old findings are most probably due to detection artifacts $[7,10]$, and that if phosphorylated PLs present at all in MGS their percentage is as low as $0.01 \%$ for most mammals (including human and steers). However when PLs are artificially added to meibium as in our experiments or when lipid-containing artificial tears are supplemented to the eye during therapy, the resulting conditions correspond well with the ones presumed by McCulley and Shine. In their model PLs form monolayer at the air/water interface on the top of which spreads the bulky hydrophobic fraction of MGS. Thus PL monolayer bridges the hydrophobic nebula with the polar water subphase and its acyl chain region provides a non-polar environment in which some of the meibium hydrophobic compounds partially solubilize. Possible interpretation of our results might be related with the structure of pure SM and pure DPPC monolayers at the air/water interface. It is well known that SM films in contrast to DPPC ones lack long range order and have amorphous structure [43]. This correlates well with the lower maximal values of reciprocal compressibility achieved by
SM monolayers when compared to DPPC films as observed in our work and in previous studies [29,34]. The nearest neighbor approximation [44] relates the lower order of SM films with the fact that self-associative enrichment of sphingolipids is actually thermodynamically disfavored which is also confirmed experimentally by calorimetric and monolayer studies [45]. In biomembranes SMs are known to aggregate together with free sterols in highly structured 2D condensates called lipid rafts [34]. However in his recent study [46] Butovich demonstrated that the percentage of free sterols in human meibium is extremely low (about $0.5 \%$ ). In our experiments with pure MGS films, we tested the presence of free sterols at the air/water interface by injecting $\beta$-cyclodextrines (known to uptake sterol molecules) in the water subphase with subsequent registration of the resulting film area loss at constant surface pressure as described in [47]. However no area changes occurred even after few hours expectation (data not shown) which strongly suggests that there are no free sterols located at the interface in the studied films. Thus it is possible that SM forms more disordered film than DPPC between the water subphase and the MGS compounds located on top (Fig. 11).

In such sparser monolayer the meibium molecules (both polar and non-polar) might incorporate more readily, which can result into significant reduction of the aggregation and thickness of the MGS “islands" especially when SM is in excess. Such interpretation correlates well with the data that SM might solubilize triglycerides [48], one of the major hydrophobic compounds of MGS, in up to 4.5 $\mathrm{mol} \%$ and in the resulting mixed films the triglycerides might act as a surfactant molecules with their carbonyl groups oriented towards water [49]. In contrast the solubility of triglycerides in PC films is reported [50] to be much lower ( $<2.8 \mathrm{~mol} \%)$. Also DPPC is known to form highly condensed films when mixed with variety of fatty acids. This might account for the lipid aggregation in thick domains observed in our experiments as some studies suggest that complex fatty acids might be the actual polar surfactant of meibium [7,51]. In addition it has been demonstrated that films with high DPPC content tend to "squeeze out" of the air/water interface less surfaceactive molecules during compression [26]. Our results are in good agreement with the results of Korb and others [11-17] that artificial tears containing phosphatidylcholine and other phospholipids strongly increase the thickness and the non-invasive break-up time of TF in vivo.

The difference in the interactions of SM and DPPC with meibium is also reflected by the different capability of the two PLs to increase the maximal reciprocal compressibility of the mixed films with the effect of DPPC being stronger than the one of SM for the whole tested range of PL/MGS ratios. Despite of the condensed appearance of MGS islands as viewed by us and by other researchers [20] the values of the elastic moduli of pure MGS films (maximal $C_{s}^{-1}$ value $=47 \mathrm{mN} / \mathrm{m}$ ) remains below $100 \mathrm{mN} / \mathrm{m}$, i.e. according the classification of Davies and Riedel meibium behaves like a monolayer liquid-expanded phase. The result confirms the measurements of Millar et al. [20] of very high lateral diffusion of fluorescently labelled molecules in meibium films. These results also might be explained by the bulky structure of MGS aggregates (multilayer or thick oily phase with low order) at the air/water interface as $C_{s}^{-1}$ of 3D surface aggregates tends to be low [52].

The increase of $C_{s}^{-1}$ values caused by the polar lipids agrees well with the predictions [10] that PL should "solidify" meibium if present in substantial amounts. Both PL also increased the maximal surface pressures achieved by the pseudo-binary films during compression. The tear film lipid layer is the key compound responsible for the surface tension of the natural tears $[4,5,8]$. The increase of its capability to reach high surface pressures (i.e. low surface tensions) should be beneficial for TF stability in vivo, as numerical simulations demonstrate that lower tear surface tension should result in prolonged TF non-invasive break-up time [2,3]. Experimental studies 


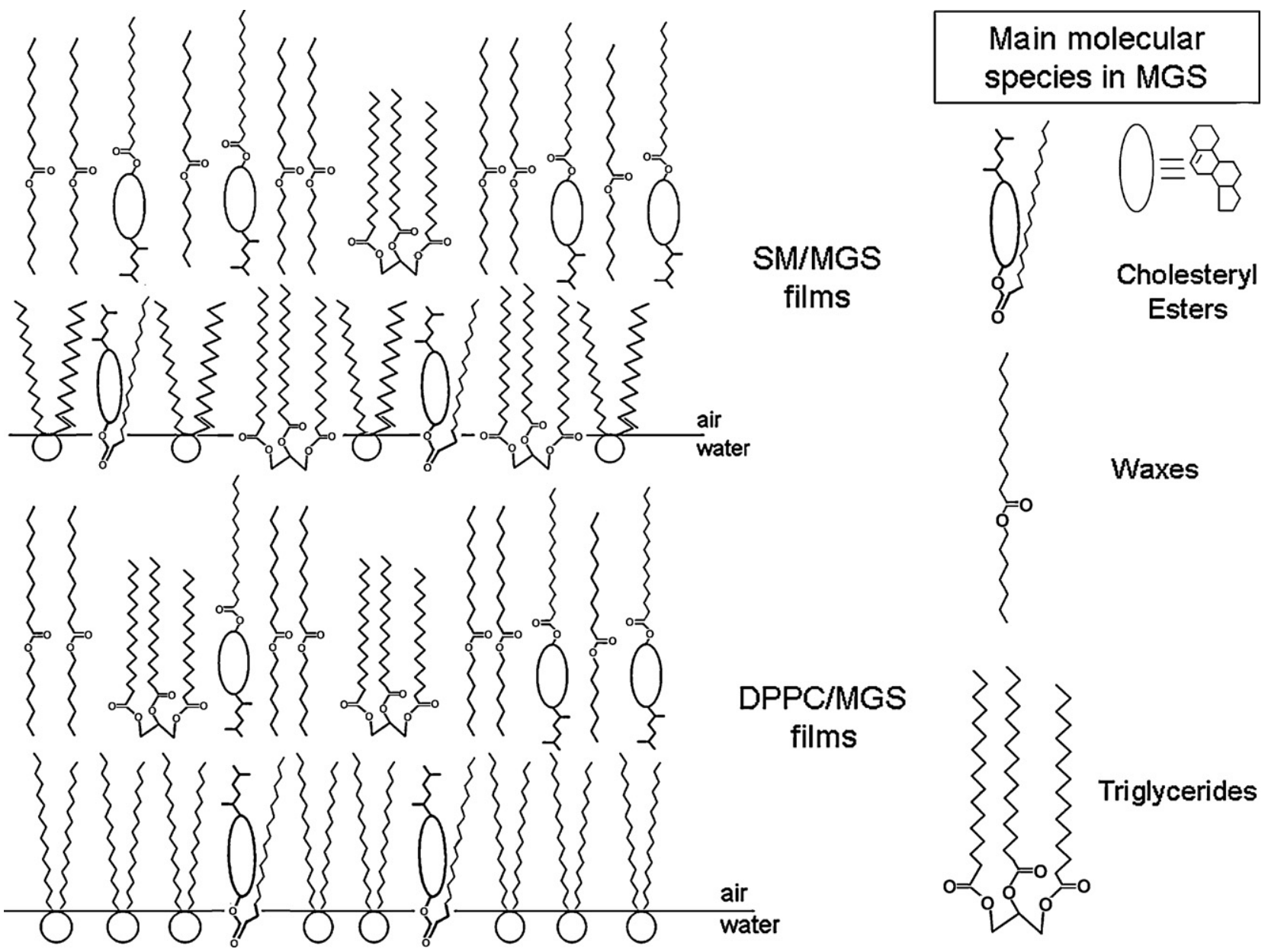

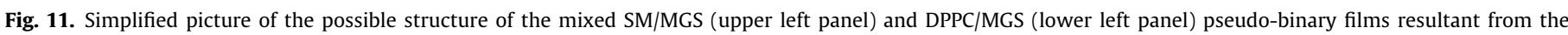

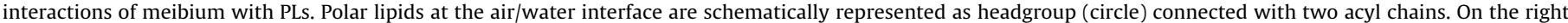

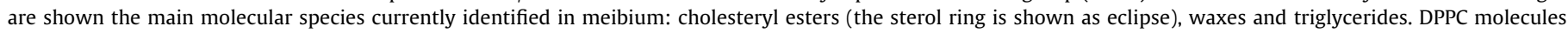

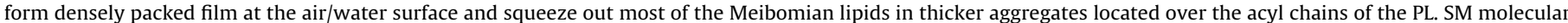

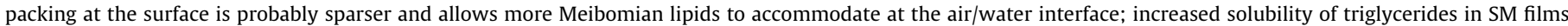

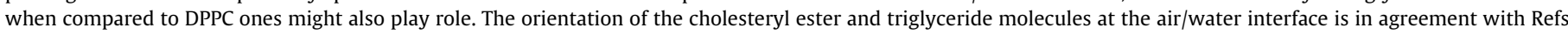
$[4,5,50]$. See Section 4 for details.

also reported that the equilibrium and maximal surface pressures achieved by tears from "healthy eyes" (where TF is stable for more than $15 \mathrm{~s}$ ) is about $5 \mathrm{mN} / \mathrm{m}$ higher than the surface pressures of samples from "dry eyes" (where TF ruptures after less than $10 \mathrm{~s}$ ) $[8,28]$. Again DPPC has more profound influence on $\pi_{\max }$ than SM, particularly in compositions with low PL amount. The dependence of $C_{s}^{-1}$ and $\pi_{\max }$ on PL type and content agrees well with the idea for domain aggregation taking place in DPPC/MGS mixtures that results in formation of more solid interfacial structures. Numerous studies of lung surfactant [26 and references there in] have demonstrated identical trend: that compared to other phosphoand sphingolipids, DPPC is of major importance for the formation of films able to attain high surface pressure during dynamic area compression. That is essential for the proper function of lung surfactant films in vivo as it makes them non-collapsible under physiological conditions. Increased resistance to collapse might be of importance for tear film functionality too, as TF should resist to dramatic and rapid area changes during blink. The stronger impact of DPPC on $C_{s}^{-1}$ and $\pi_{\max }$ of the pseudo-binary films correlates with the old works of Tiffany [8] demonstrating that phosphatidylcholines are best amongst the polar lipids in lowering the surface tension of "delipidated tears" (the aqueous fraction obtained after chloroform extraction of intact tears) and with the results of Korb et al. [11] that amongst range of zwitterionic phospholipids, DPPC produces longest increase of non-invasive break-up time when instilled in TF in vivo. Thus by coupling the measured impact of PL on tear film stability in vivo to the effect of PLs on the reciprocal compressibility, maximal surface pressures and the structure of pseudo-binary $\mathrm{PL} / \mathrm{meibium}$ films in vitro, it might be possible to obtain molecular scale insights how polar lipids achieve their function at the ocular surface.

The dynamic isocycling does not change significantly the shape of the $\pi-A$ isotherms of the studied films. All films show low $\pi-A$ hysteresis. For DPPC/MGS films the hysteresis is somewhat lower than for the rest of the studied compositions which might be beneficial in vivo concerning the rapid rate of the eyelid movement during blinking.

There is contradiction in the existing literature concerning the presence of PLs in the surface layer of TF and their role in TF lipid layer formation and/or disintegration. The old findings that phospho- and sphingolipids comprise up to $10 \%$ of MGS are now attributed to detection artifacts [7,10]. However it is possible tear film PL to originate from sources like aqueous tears, cornea, and conjunctiva $[9,10]$. Thus at the current moment there are no data about the ratio between PL and MGS at the air/tear interface. Considering the massive amount of non-polar lipid secretions produced by Meibomian glands it is realistic to suggest that MGS is in excess to PL at the ocular surface. Thus it is hard to judge about the phys- 
iological relevance of our results in relation to the normal tear film. The minimal amount at which PLs significantly alter the surface properties of MGS in our experiments is 0.2 molar parts. We selected SM and DPPC as they were already used as synthetic mimics of tear PLs [24,25]. However Blanksby [53] recently reported that although PC and SMs are abundant in tear's aqueous layer, their acyl tails are longer than C16 and (poly)-unsaturated which will be of great impact for their intermolecular interactions with other lipids. Also it is important to test the interaction of PLs with human MGS which will be task of our future study.

\section{Conclusions}

Despite of the uncertainties regarding the content and the composition of PLs in natural TF, the polar lipid type and amount at the ocular surfaces can be readily altered in broad range by the application of lipid-containing artificial tears. In the current work we demonstrate that PLs are capable to interact with MGS with different strength and in different manner (resulting in aggregation or disaggregation of the thick meibium islands) and to increase the mixed films reciprocal compressibility and capability to achieve higher surface pressures. The results correlate well with the capacity of polar lipid-containing artificial tears to increase the stability of tear film in vivo. Thus applying the techniques of surface chemistry, like surface balance measurements, might be very useful for studying the interactions of MGS with pharmaceutically applicable PLs on a molecular level and for the selection of desired molecules that can be further used for the development of drug compositions.

\section{Acknowledgments}

Authors gratefully acknowledge the financial support of this study provided by contract DO $02-280 / 2008$ and partially by contract DO 02-107/2008 funded by Bulgarian Ministry of Education and Science. We are also thankful to Prof. R. Ivanov (Department of Animal and Human physiology, Faculty of Biology, Sofia University) for the help in collecting the MGS samples and to Dr. Aapi Sinkkanen (Kibron Inc., Finland) for the strong technical support of our research. We are especially obliged to Prof. Christian Vassillieff (Department of Physical Chemistry, Faculty of Chemistry, Sofia University) for the numerous discussions and advices during the accomplishment of the study and the preparation of the manuscript.

\section{References}

[1] E. King-Smith, B. Fink, R. Hill, K. Koelling, J. Tiffany, Curr. Eye Res. 29 (2004) 357.

[2] A. Sharma, S. Tiwari, R. Khanna, J.M. Tiffany, Adv. Exp. Med. Biol. 438 (1998) 425.

[3] H. Wong, I. Fatt, C.J. Radke, J. Colloid Interface Sci. 184 (1996) 44.

[4] J.P. McCulley, W. Shine, Trans. Am. Ophthalmol. Soc. 95 (1997) 79.

[5] J.P. McCulley, W.E. Shine, Biosci. Rep. 21 (2001) 407.

[6] I.A. Butovich, T.J. Millar, B.M. Ham, Curr. Eye Res. 33 (2008) 405.

[7] I.A. Butovich, Prog. Ret. Eye. Res. (2009) 1-16, doi:10.1016/j.preteyeres.2009. 07.002 .
[8] B. Nagyová, J.M. Tiffany, Curr. Eye Res. 19 (1999) 4.

[9] D. Borchman, G.N. Foulks, M.C. Yappert, D. Tang, D.V. Ho, Chem. Phys. Lipids 147 (2007) 87.

[10] I.A. Butovich, Invest. Ophthalmol. Vis. Sci. 49 (2008) 3779.

[11] D.R. Korb, J.V. Greiner, T. Glonek, Adv. Exp. Med. Biol. 506 (2002) 495.

[12] S. Lee, S. Dausch, G. Maierhofer, D. Dausch, Klin. Monatsbl. Augenheilkd. 221 (2004) 825.

[13] D.R. Korb, R.C. Scaffidi, J.V. Greiner, K.R. Kenyon, J.P. Herman, C.A. Blackie, T Glonek, C.L. Case, V.M. Finnemore, T. Douglass, Optom. Vis. Sci. 82 (2005) 594.

[14] M.A. Lemp, G.N. Foulks, U. Devgan, W.B. Trattler, K.K. Nichols, Refract. Eyecare Ophthalmol. 9 (Suppl.) (2005) 3-15.

[15] D. Dausch, S. Lee, S. Dausch, J.C. Kim, G. Schwert, W. Michelson, Klin. Monatsbl. Augenheilkd. 223 (2006) 974.

[16] R.C. Scaffidi, D.R. Korb, Eye Contact Lens 33 (2007) 38.

[17] R. Khaireddin, K.G. Schmidt, Klin. Monatsbl. Augenheilkd. (2009), doi:10.1055/s-0028-1109686, Epub ahead of print.

[18] F. Miano, M. Calcara, T.J. Millar, V. Enea, Colloids Surf. B: Biointerfaces 44 (2005) 49.

[19] S.T. Tragoulias, P.ip J. Anderton, G.R. Dennis, F. Miano, T.J. Millar, Cornea 24 (2005) 189.

[20] T.J. Millar, S.T. Tragoulias, P.J. Anderton, M.S. Ball, F. Miano, G.R. Dennis, P. Mudgil, Cornea 25 (2006) 91.

[21] P. Mudgil, M. Torres, T.J. Millar, Colloids Surf. B: Biointerfaces 48 (2006) 128.

[22] P. Mudgil, T.J. Millar, Exp. Eye Res. 86 (2008) 622.

[23] T.J. Millar, P. Mudgil, I.A. Butovich, C.K. Palaniappan, Invest. Ophthalmol. Vis. Sci. 50 (2009) 140.

[24] K. Peters, T. Millar, Curr. Eye Res. 25 (2002) 55.

[25] P.G. Petrov, J.M. Thompson, I.B. Abdul Rahman, R.E. Ellis, E.M. Green, F. Miano, C.P. Winlove, Exp. Eye Res. 84 (2007) 1140.

[26] O. Blanco, J. Pérez-Gil, Eur. J. Pharmacol. 568 (2007) 1.

[27] N. Nicolaides, J.K. Kaitaranta, T.N. Rawdah, J.I. Macy, F.M. Boswell, R.E. Smith, Invest. Ophthalmol. Vis. Sci. 20 (1981) 522.

[28] J. Zhao, P. Wollmer, Clin. Physiol. 21 (2000) 282.

[29] K. Sabatini, J.-P. Mattila, P.K.J. Kinnunen, Biophys. J. 95 (2008) 2340.

[30] H.L. Brockman, Chem. Phys. Lipids 73 (1994) 57.

[31] F.C. Goodrich, in: J.H. Schulman (Ed.), Proc. Second International Congress on Surface Activity, vol. I, Butterworth \& Co., London, 1957, p. 85.

[32] N. Gershfeld, Intermolecular energies in condensed, lipid monolayers on water, J. Colloid Interface Sci. 32 (1970) 167.

[33] A. Fernandez-Botello, F. Comelles, M. Asuncion Alsina, P. Cea, F. Reig, J. Phys. Chem. B 112 (2008) 13834.

[34] J.M. Smaby, V.S. Kulkarni, M. Momsen, R.E. Brown, Biophys. J. 70 (1996) 868.

[35] J.T. Davies, E.K. Rideal, Interfacial Phenomena, 2nd ed., Academic Press, New York, 1963, p. 265.

[36] H.M. Mansour, G. Zografi, Langmuir 23 (2007) 3809.

[37] E. Prenner, G. Honsek, D. Hönig, D. Möbius, K. Lohner, Chem. Phys. Lipids 145 (2007) 106.

[38] F.G. Shellock, C.J. Schatz, Radiology 185 (1992) 697.

[39] P.B. Morgan, A.B. Tullo, N. Efron, Eye 9 (1995) 615.

[40] P. Mudgil, Proc. of Solving Dry Eye: An International Exchange Solving Dry Eye: An International Exchange, Sydney, 3-8 August, 2009, p. p.12.

[41] W.E. Shine, J.P. McCulley, Arch. Ophthalmol. 116 (1998) 849.

[42] W.E. Shine, J.P. McCulley, Curr. Eye Res. 26 (2003) 89.

[43] D. Vaknin, M.S. Kelley, J. Chem. Phys. 115 (2001) 7697.

[44] S.L. Regen, Curr. Opin. Chem. Biol. 6 (2002) 729.

[45] B. Maggio, Prog. Biophys. Mol. Biol. 62 (1994) 55.

[46] I.A. Butovich, E. Uchiyama, M.A. Di Pascuale, J.P. McCulley, Lipids 42 (2007) 765.

[47] H. Ohvo, J.P. Slotte, Biochemistry 35 (1996) 8018.

[48] I. Arimoto, H. Saito, Y. Kawashima, K. Miyajima, T. Handa, J. Lipid Res. 39 (1998) 143.

[49] G. Hennere, P. Prognonb, F. Brionc, I. Nicolis, Chem. Phys. Lipids 157 (2009) 86.

[50] J.A. Hamilton, D.M. Small, Proc. Natl. Acad. Sci. U.S.A. 78 (1981) 6878.

[51] K.K. Nichols, B.M. Ham, J.J. Nichols, C. Ziegler, K.B. Green-Church, Invest. Ophthalmol. Vis. Sci. 48 (2007) 34.

[52] A.W. Adamson, A.P. Gast, Physical Chemistry of Surfaces, 6th ed., John Wiley \& Sons, New York, 1997.

[53] S. Blanksby, Proc. of Solving Dry Eye: An International Exchange Solving Dry Eye: An International Exchange, Sydney, 3-8 August, 2009, p. 18. 